\title{
Studying the Atomic Structures by Aberration-Corrected and Conventional Electron Microscopy
}

\author{
Y. M. Wang
}

1. Beijing National Laboratory for Condensed Matter Physics, Institute of Physics, Chinese Academy of Sciences, Beijing, China

Image resolution is mainly limited by the spherical aberration $(\mathrm{Cs})$ of the objective lens. There are two effective approaches to correct the Cs. One is the hardware aberration correction, so- called Cscorrector [1]. The other is software method including electron crystallographic image processing [2-4] and exit wave reconstruction $[5,6]$. With a Cs-corrector, the image resolution can be extended to subangstrom level such that oxyzen atoms can be relatively easily detected by the negative Cs imaging technique [7] and lithium atoms indentified by annular-bright-field (ABF) image[8]. By software method, the light atoms such as lithium [9], were directly resolved from HRTEM images by the exit wave reconstruction. Using two-stage electron crystallographic image processing[4] -image deconvolution and phase extension the light atoms as oxygen and boron atoms [10] have been resolved from a single image.

In the present paper, we will show the results in atomic-scale structure investigations using the software and hardware aberration correction, respectively. For software coorection, we take advantage of the two-stage image processing technique, using the iron-based superconductor SmFeAsO0.85F0.15 as an example, to improve the image resolution. Compared with other method in HRTEM, the approach used here only need one image collected at arbitrary defocus value rather than a series of through focus images. Image deconvolution as a special kind of image processing in HRTEM is used to restore the image distortion due to the lens aberrations. More accurately, it aims at transforming an image not representing the crystal structure intuitively into the structure projection, the resolution of which is limited by the information limit of the microscope. Electron diffraction unrestricted by lens aberrations could overcome this resolution limit. The reachable imag resolution is better than $1 \AA$ using a combination of the electron diffraction and the image deconvolution. By this appraoch, the atomic columns of $\mathrm{O}$ and considerably heavier $\mathrm{Sm}$ at a very close distance $(1.17 \AA)$ in $\mathrm{SmFeAsO} 0.85 \mathrm{~F} 0.15$ are simultaneously revealed from a single image taken with a conventional $200 \mathrm{kV}$ electron microscope [11]. It is for the first time that the $\mathrm{O}$ atoms adjacent to the heavier atoms at a so close distance are imaged by this approach. In addtion, we will report three different types of interlocked ferroelectric-antiphase DWs and two abnormal topological four-state vortex-like domain patterns at the atomic level in multiferroic manganite YMnO3 by using the hardware correction method, i.e. the CS-corrected high angle annular dark field scanning transmission electron microscope (HAADF-STEM) imaging techniques. Recently, Cs-corrected electron microsopy, especially the STEM technique has been widely used to provide high-resolution structural information. Unlike that of conventional HRTEM images, the contrast of HAADF- STEM images is roughly proportional to $\mathrm{Z}^{1.7}$, where $\mathrm{Z}$ is the atomic number. And it is rarely affected by a small variation of the specimen thickness. Using this imaging technique, three different types of ferroelectric-antiphase DWs with a translation vector (i.e., 1/6[210] or $-1 / 6[210])$ are observed at atomic level. Then the configurations of the two abnormal topological four-state vortex-like domains with different DWs are directly determined at the 
first time, which are different from the traditional four-state vortex domain caused by edge dislocations[12]. [13]

References:

[1] M. Haider, G. Braunshausen and E. Schwan, Optik 99 (1995), p.167.

[2] F.H. Li, Phys Status Solidi A 207 (2010) p.2639.

[3] L. De Caro et al, Nat. Nano. 5 (2010), p. 36.

[4] W. Wan, S. Hovmöller and X.D. Zou, Ultramicroscopy 115 (2012), p.60.

[5] M. OpdeBeeck, D. VanDyck, Ultramicroscopy 64 (1996), p.153.

[6] J.H. Chen, H.W. Zandbergen and D. VanDyck, Ultramicroscopy 98 (2004), p.81.

[7] C.L. Jia, M. Lentzen and K. Urban, Science 299 (2003), p.870.

[8] Y. Sun et al, Nat. Commun. 4 (2013), p.1870.

[9] Y. Shao-Horn et al, Nat. Mater. 2 (2003), p.464.

[10]H.B. Wang et al, Acta Cryst. A 58 (2002), p.494.

[11]Y.M. Wang, B. H. Ge and G. Che, Micron 71 (2015), p.32.

[12]L. Tian et al, APL 106 (2015), p.112903.

[13]The author acknowledge funding from the National Natural Science Foundation of China

(Grant No. 11474329).
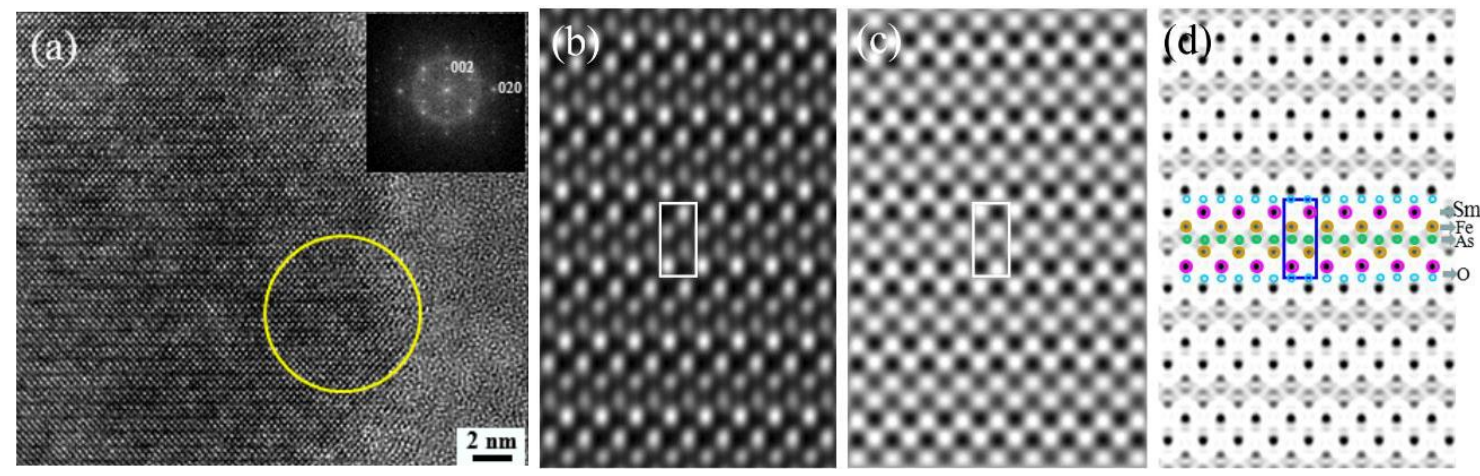

Figure 1. (a)[100] zone-axis HRTEM image, (b) and (c) Fourier filtering image and deconvoluted image, $(\mathrm{d})$

structure projection after phase extension in combination with the diffraction intensity correction.
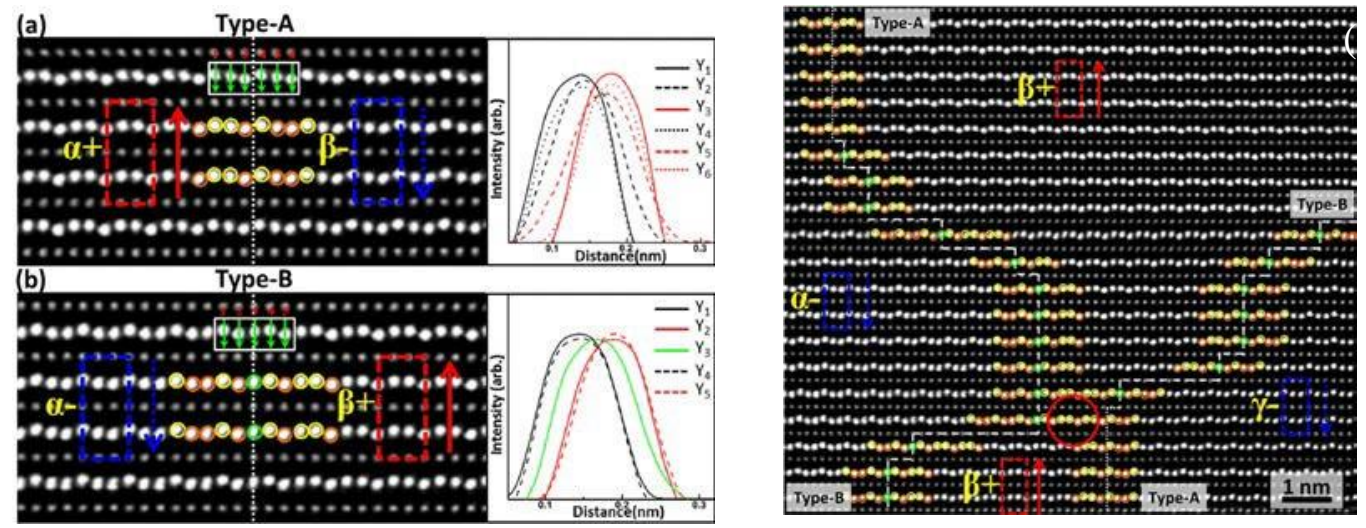

Figure 2. HADDF-STEM images for (a) Type-A, (b) Type-B interlocked ferroelectric DWs along [010] direction, and (c) for the four-state vortex-like domain. 